\title{
Second Opinion System for Intraoral Lesions
}

\author{
Orazio Gambino, Fausto Lima \\ Roberto Pirrone and Edoardo Ardizzone \\ Department of Chemical Management \\ Mechanical Engineering and Computer Science \\ University of Palermo \\ viale delle Scienze, Building 6 \\ Email: orazio.gambino,lima.fausto, \\ roberto.pirrone,edoardo.ardizzone@unipa.it
}

\author{
Giuseppina Campisi and Olga di Fede \\ Department of Surgical, Oncological \\ and Oral Sciences"Giuseppe Messina" \\ University of Palermo \\ via del Vespro \\ Email: giuseppina.campisi, olga.difede @unipa.it
}

\begin{abstract}
In this paper we present the prototype of a teledentistry system to perform the remote diagnosis of oral diseases. It makes use of a particular device called intra-oral (or dental) camera properly designed to shoot video and take pictures of the inner part of the mouth. The intra-oral cameras can be connected via USB to a common $P C$ and they are very cheap, unlike the intra-oral photography kit for DSLR cameras. Usually this kind of devices are used in dentistry studies for local visualization by means of specialized software. The novelty of our system is that the real-time video produced by this device is canalized into a video streming by means of VideoLan client/server (VLC) and pictures can be sent by a current File Tranfer Protocol (FTP) service to realize a Second Opinion System.
\end{abstract}

\section{INTRODUCTION}

Second opinion systems are often a branch of telemedicine: a mixture among telecommunication, computer science and medicine. When telecommunication and computer science meet with dentistry a new branch of telemedicine is born: the teledentistry. Thanks to teledentistry, it is possible to perform cooperative work, distant consultations, sharing digital information like images, documents, radiographies an so on. The very first project on teledentistry was a military project of the United States Army (U.S.Armys Total Dental Access Project)[1]. Many other works oriented to teledentistry can be found in literature. In [2] digital images of oral mucosal lesions are acquired by means of an intra-oral camera and stored on a personal computer; subsequently, they are transmitted via the Internet to a distant site and compared with the original ones. A pool of physicians didn't notice any loss of quality between transmitted and original images. In [3] the authors show that the intra-oral camera increases the number of occlusal lesions detected with respect to an unaided vision. In [4] a teledentistry system aimed to increase and enhance the children's oral health quality who live in rural areas of California is reported. In [5] a comparison between digital images of oral mucosal acquired with a SLR and intra-oral camera is reported. A teledentistry system to perform remote diagnosis on children while they are at school is described in [6][7]. In [8] a teledentistry system is used to the diagnosis and routine management of endodontic/oral surgery patients. A system aimed to the remote recognition of root canal orifices is tested in [9] where the images are acquired thanks to an intra-oral camera. In this paper we present a teledentistry system able to perform a video streaming, medical/radiological image sharing, and medical tele-reporting system aimed to remote first diagnosis or second opinion. Thanks to the video streaming, the physician can drive the operator during medical examination on the patient. Both images acquired with intra-oral camera and radiographic images can be transmitted by the operator even if the physician is not logged. The system uses low cost equipment and doesn't require a particular internet connection: it can use a common ADSL line or Wi-Fi connection. The paper is organized as follows: Section 2 describes the system, Section 3 shows its use, in Section 4 some conclusion are written.

\section{BRIEF SYSTEM DESCRIPTION}

For this project, the intra-oral camera HK-790 has been chosen. We chosen this device because it is the only intraoral camera equipped with a 5.0 Megapixel sensor so that still images have a resolution of $2560(\mathrm{H}) \times 1920(\mathrm{~V})$ and, on the other hand, its price is very cheap. Indeed, its price is around 200 euros, unlike most of the other cameras which offer lower features with a price over the 1000 euros and more. Unfortunately, the first device exhibited some damaged pixels, so we returned it. The new camera is in order but, in correspondence of some particular orientations, the Leds sites are projected on the image like dark circles, as shown in fig. 1. The artifact just mentioned arise during the streaming visualization, while it doesn't appear for the still image mode. At the end, the quality/price ratio is good and it can be used for this purpose. The actors of the system and their roles are summarized in fig.2. Briefly, the administrator performs the management of the system, the doctor makes the diagnosis on the basis of data received by the operator performing the medical examination. Two modes are supported by the system: the Online Mode and Offline Mode. The former is active when both the physician and the operator are logged to the system and it allows to perform a real-time medical examination via video streaming. The acquired images can be sent by the operator to the patient's specific folder and they are immediately available to the physician who can give other instructions to the operator to perform new acquisitions. The latter mode is active when only the operator or only the physician is logged to the system. In both of cases, the realtime video streaming is not active. If only the operator is logged, he/she can send the acquired images to a temporary folder and a request is sent to the physician web page. In this way, the operator can perform a medical examinations session for many patients and the physician can view the data and write the medical report in a second time. If only the 
physician is online, he/she will find the requests previously sent by the operator. There will be a request for each patient. The physician can select a request and a web page with an image gallery will be shown. The physician can select the more interesting images to include in the medical report.
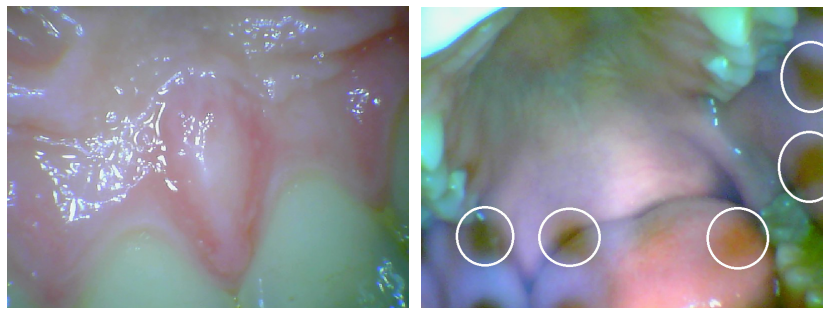

Fig. 1. Image acquisition with the intra-oral camera HK-790. LEFT: an image acquired without any artifact. RIGHT: White circles put in evidence some artifacts introduced by the intra-oral camera during the streaming mode.

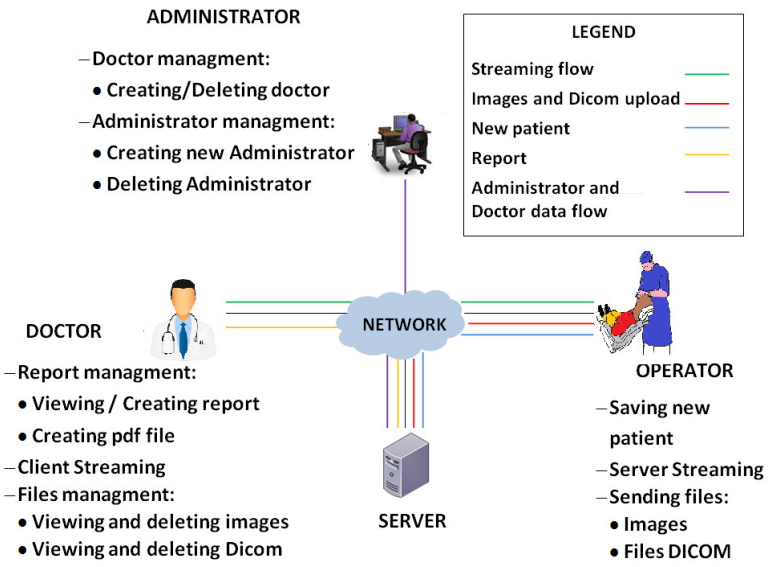

Fig. 2. System Architecture Schema.

\section{USE OF PROTOTYPE}

As shown in the pictures in fig.3, the system investigated allows to recognize exactly, both out or inside the oral cavity, characteristics such as site, color (e.g. red and white variants) and surface (homogeneous vs non-homogeneous, verrucous, proliferative, and so on) of a given lesion. From the latest features, both directly (classic way) and remotely (modern alternative), it is possible to make a provisional diagnosis in the majority of the cases, a definitive one in some cases (e.g. true reticular lichen planus, without any further confirming surgical procedure), and surely to provide, in all cases, an optimal clinical second opinion.

\section{CONCLUSIONS AND FUTURE WORKS}

A teledentistry system aimed to second opinion has been presented in this paper. It is a low cost system and doesn't need particular internet connection. It allows to perform a medical examinations session on the patients also when the physician is not logged. A better intra-oral camera will be used for future versions of this system: even if other manufacturers provide intra-oral cameras whose features are lower than the one used in this work, we will investigate on the use of the real-time video streaming without any artifact introduced by

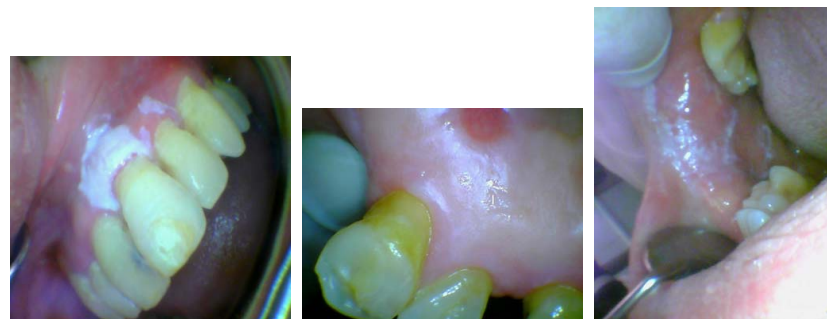

Fig. 3. a) Oral leukoplakia on gingiva of upper incisors; b) Provisional remote diagnosis, thanks to telemedicine, of a potentially malignant lesion of palatal mucosa, a non-homogeneous (white and red) form of lichenoid lesion. c) Definitive clinical remote diagnosis, thanks to telemedicine, of a true reticular lichen planus, in vestibular and retro-commissural mucosal area

the device. A better integration of the intra-oral camera with the system will be made in cooperation with the research and development department of the intra-oral camera manufacturer. Actually, the real-time video streaming is performed using mepg4 format, which performs a lossy compression but the medical quality is good to recognize heavy oral diseases. We will investigate the possibility to perform a transmission using the h264 format, without any compression, and for this reason a dedicated line will be requested to the internet provider, especially for the operator terminal ad in particular for the upload mode. Moreover, the video streaming will be recorded in such a way that the physician can also see the video of a medical examination like the other data.

\section{ACKNOWLEDGMENT}

The authors express their gratefulness to the Rotary Club Palermo Teatro del Sole for the financial support given to this work.

REFERENCES

[1] Vandre, R.H. ; Kudryk, V.L. ; Fay, C.R., III ; Edwards, J.C., Jr. ; Jones, T.K. US Army teledentistry Proceedings of the National Forum Military Telemedicine On-Line Today, 1995. Research, Practice, and Opportunities.,DOI: 10.1109/MTOL.1995.504530 pp 53-56 (1995)

[2] Leao JC, Porter SR. Telediagnosis of oral disease Brazilian Dental Journal vol.10 n.1 pp: 1-60 ISSN 0103-6440 (1999)

[3] A.H. Forgie, C.M. Pine, N.B. Pitts The assessment of an intra-oral video camera as an aid to occlusal caries detection International Dental Journal Volume 53, Issue 1, pages 36, February 2003

[4] Chang SW, Plotkin DR, Mulligan R, Polido JC, Mah JK, Meara JG.teledentistry in rural California: a USC initiative. Journal of the California Dental Association. Aug;31(8):601-8. 2003

[5] R. N. Smith, A. Rawlinson, D. L. Lath, A.H. Brook. A digital SLR or intra-oral camera: preference for acquisition within an image analysis system for measurement of disclosed dental plaque area within clinical trials Journal of Periodontal Research Volume 41, Issue 1, pages 5561, February 2006

[6] Kopycka-Kedzierawski, DT and Billings, RJ.teledentistry in innercity child-care centres Journal of Telemedicine Telecare. Vol.12, No.4,pp.176-81, ISSN 1357-633X. (2006)

[7] Berndt J, Leone P, King G. Using teledentistry to provide interceptive orthodontic services to disadvantaged children American Journal of Orthodontics and Dentofacial Orthopedics vol.34 n.5 pp. $700-706$ (2008)

[8] Zivkovic D., Tosic G., Mihailovic B., Miladinovic M., Vujicic B.Diagnosis of periapical lesions of the front teeth using the internet PONS Medical Journal, Volume 2010; vol.7 n.4- pp 138143 ISSN:18202411

[9] Brllmann D, Schmidtmann I, Warzecha K, and d'Hoedt B, Recognition of root canal orifices at a distance - a preliminary study of teledentistry Journal Of Telemedicine And Telecare, vol. 17, no. 3, pp. 154-157. (2011) 